\title{
Mathematische Modellierung elektrokatalytischer Zellen
}

\author{
Manuel Landstorfer
}

\begin{abstract}
Elektrokatalytische Reaktionen sind von zentraler Bedeutung für eine Vielzahl moderner Technologien und Prozesse, unter anderem für Brennstoffzellen, Elektrolyse-Verfahren, Galvanik, Sensorik oder Batterien. Die (Weiter-)Entwicklung elektrokatalytischer Zellen hinsichtlich ihrer Kosten, Effizienz und Haltbarkeit, aber auch ihrer Umweltverträglichkeit ist weltweit ein immenses Forschungsfeld. Mathematischen Modelle, die im Rahmen von MATH+ entwickelt werden, helfen durch (computergestützte) Vorhersagen bei dieser Entwicklung. Zentral dabei ist eine konsistente und zuverlässige Modellierung um eine echte Vorhersagekraft bzgl. verschiedener Parameter-Variationen gewährleisten zu können.
\end{abstract}

Eine elektrochemischen Zelle besteht allgemein aus einer (porösen) Kathode K, einem Elektrolyt E, und einer (porösen) Anode A. Reagiert an der Grenzfläche K|E z. B. ein Elektron $\mathrm{e}^{-}$aus der Kathode $\mathrm{K}$ mit einem Kation $\mathrm{C}^{+}$aus dem Elektrolyt $\mathrm{E}$ zu einem Reaktionsprodukt D im Elektrolyt E, so nennen wir diesen Prozess elektrokatalytische Reaktion und schreiben

$$
\left.\mathrm{C}^{+}\right|_{\mathrm{E}}+\left.\left.\mathrm{e}^{-}\right|_{\mathrm{K}} \rightleftharpoons \mathrm{D}\right|_{\mathrm{E}}
$$

Aufgrund der beteiligten Elektronen in (1) fließen weitere Elektronen $\mathrm{e}^{-}$durch den äußeren Stromkreis von Anode zu Kathode nach und können über ein Strommessgerät detektiert werden. Über den gemessenen Strom $I$ lassen sich dann Rückschlüsse über die Reaktionsrate von (1) gewinnen, insbesondere wie stark ein spezifisches Kathodenmaterial die Reaktion (1) katalysiert. Die Reaktion (1) verbraucht aber nicht nur Elektronen $\mathrm{e}^{-}$, es werden auch Kationen $\mathrm{C}^{+}$verbraucht und Reaktionsprodukte D erzeugt, die ihrerseits jeweils an- bzw. abtransportiert werden müssen. Diese Transportprozesse haben einen wesentliche Einfluss auf den Wirkungsgrad der Zelle und müssen in einem elektrochemischen Modell entsprechend berücksichtigt werden.

Im Gegensatz zu einem normalen Kondensator, der ebenfalls aus Anode und Kathode aufgebaut ist, erzeugt die
Reaktion (1) in der elektrochemischen Zelle eine Zellspannung $U \neq 0$, die wiederum abhängig ist von der Stromstärke $I$. Das nichtlineare Wechselspiel zwischen Strom $I$ und Spannung $U$ ist in einer elektrochemischen Zelle weitaus komplexer als in inaktiven elektrischen Bauteilen. Charakteristisch für die Prozesse in einer elektrochemischen Zelle ist die Strom-Spannung-Kennlinie, bei der die Zellspannung $U$ gegen den äußeren Strom $I$ oder gegen $Q=\int_{0}^{t} I d t^{\prime}$ aufgetragen und experimentell bestimmt wird. Die Validierung eines mathematischen Modells geschieht daher in der Regel über berechnete $(I-U)$-Kennlinien.

Die Abbildung der verschiedenen Prozesse einer elektrokatalytischen Zelle in ein mathematisches Modell geschieht mithilfe der Kontinuumsmechanik. Hierbei wird den Teilchensorten $\mathrm{C}^{+}, \mathrm{e}^{-}$, D eine Teilchendichte $n_{Z}(\mathbf{x}, t), Z \in$ $\{C, e, D\}$ zugeordnet, wobei $\mathbf{x} \in \Omega \subset$ die Ortsvariable und $t \in I \subset \mathbb{R}$ die Zeit ist. Diese Dichte gehorcht einer Bilanzgleichung

$$
\frac{\partial n_{Z}(\mathbf{x}, t)}{\partial t}=-\operatorname{div} \mathbf{J}_{Z} \quad Z \in\{C, e, D\}
$$

wobei $\mathbf{J}_{Z}$ der Fluss der Teilchensorte $Z$ ist. An der Grenzfläche zwischen Kathode K und Elektrolyt E werden Randbe-

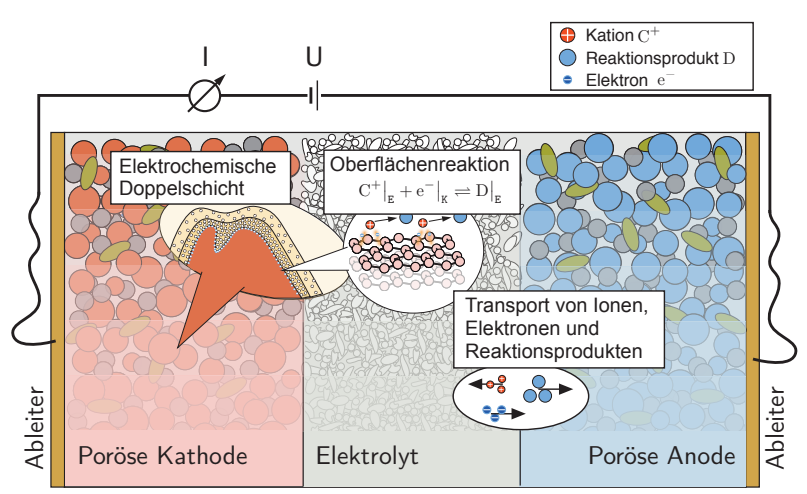

Abbildung 1. Allgemeiner Aufbau einer elektrochemischen Zelle 
dingungen für die Reaktion (1) formuliert,

$$
\left.\mathbf{J}_{C} \cdot \mathbf{n}\right|_{\mathrm{K} \mid \mathrm{E}}=-\left.\mathbf{J}_{e} \cdot \mathbf{n}\right|_{\mathrm{K} \mid \mathrm{E}}=-\left.\mathbf{J}_{B} \cdot \mathbf{n}\right|_{\mathrm{K} \mid \mathrm{E}}=\underset{s}{R},
$$

wobei $\underset{s}{R}$ die (Oberflächen-)Reaktionsrate ist. Die Herleitung eines expliziten Zusammenhangs zwischen den Flüssen $\mathbf{J}_{Z}$, der Reaktionsrate $R_{s}$, und den Teilchendichten $n_{Z}$ geschieht mithilfe der Nicht-Gleichgewichts-Thermodynamik [3]. Dieser Ansatz garantiert, dass der sogenannte 2. Hauptsatz der Thermodynamik immer erfüllt ist und das Modell somit thermodynamisch konsistent ist. Beispielsweise ist der Kationen-Fluss [1]

$$
\mathbf{J}_{C}=\mathbf{D}_{C} \cdot \Gamma^{\mathrm{tf}} \cdot \nabla n_{C}+e_{0} \mathbf{D}_{C} n_{C} \cdot \nabla \varphi,
$$

wobei $\mathbf{D}_{C}$ der Diffusionskoeffizient, $\Gamma^{\mathrm{tf}}=\Gamma^{\mathrm{tf}}\left(n_{C}\right)$ der (nichtlineare) thermodynamische Faktor, $e_{0}$ die Elementarladung und $\varphi$ das elektrostatische Potential sind. Eine ähnliche Beziehung gilt auch für die Flüsse der Elektronen $\mathrm{e}^{-}$und Reaktionsprodukte $D$. Die Reaktionsrate $R_{s}$ ist beispielsweise gegeben durch [2]

$$
\begin{aligned}
\underset{s}{R}=-\underset{s}{L} \cdot\left(\left(\frac{n_{C}^{\mathrm{E}}}{n_{D}^{\mathrm{E}}}\right)^{\alpha} \mathrm{e}^{\alpha \cdot \frac{e_{0}}{k_{\mathrm{B}} T}\left(E^{0}+\varphi^{\mathrm{K}}-\varphi^{\mathrm{E}}\right)}\right. \\
\left.\quad-\left(\frac{n_{C}^{\mathrm{E}}}{n_{D}^{\mathrm{E}}}\right)^{-(1-\alpha)} \mathrm{e}^{-(1-\alpha) \cdot \frac{e_{0}}{k_{\mathrm{B}} T}\left(E^{0}+\varphi^{\mathrm{K}}-\varphi^{\mathrm{E}}\right)}\right)
\end{aligned}
$$

mit Konstanten $\underset{s}{L}, E^{0}$ und $\alpha \in[0,1]$. Die Abkürzung $u^{\mathrm{E}}$ bedeutet eine Auswertung von $u(\mathbf{x}, t)$ an der Grenzfläche K|E aber im Elektrolyt, $u^{\mathrm{E}}=\left.u\right|_{\mathrm{K} \mid \mathrm{E}} ^{+}$, und entsprechend $u^{\mathrm{K}}$ eine Auswertung an der Grenzfläche aber in der Kathode, $u^{\mathrm{K}}=\left.u\right|_{\mathrm{K} \mid \mathrm{E}} ^{-}$ Die Größe $\varphi^{\mathrm{K}}-\varphi^{\mathrm{E}}$ beschreibt die Potentialdifferenz durch die sogenannte elektrochemische Doppelschicht (siehe Abbildung 1), deren konsistente Einbettung in die Modellierung elektrochemischer Systeme wesentlich am WeierstraßInstitut für Angewandte Analysis und Stochastik (WIAS) in den letzten Jahren voran getrieben wurde.
Im nächsten Schritt der Modellierung wird berücksichtigt, dass Kathode und Anode poröse Medien sind. Die numerische Berechnung von Gleichung (4) mit Randbedingung (5) für realistische Zellen in $3 \mathrm{D}$ ist extrem Rechenaufwendig und nicht unbedingt notwendig. Ein eleganter mathematischer Ansatz zur Beschreibung poröser Medien ist die Annahme, dass das Medium periodisch aus Einzelzellen aufgebaut ist, wobei das Längenverhältnis $\varepsilon$ zwischen Elektrode und Einzelzelle klein ist. Durch die Einführung von zwei Größenskalen, $\mathbf{x}$ für die Makroskala und $\mathbf{y}=\frac{\mathbf{x}}{\varepsilon}$ für die Mikroskala, können die Teilchendichten asymptotisch in den zwei Größenskalen entwickelt werden [4], z. B.

$$
n_{C}(\mathbf{x}, t)=n_{C}^{0}(\mathbf{x}, t)+\varepsilon n_{C}^{1}(\mathbf{x}, \mathbf{y}, t)+\mathcal{O}\left(\varepsilon^{2}\right) .
$$

Der Grenzübergang $\varepsilon \rightarrow 0$ führt dann zu sogenannten homogenisierten Bilanzgleichungen, z. B. für $n_{C}$ in der Kathode

$$
\frac{\partial n_{C}^{0}(\mathbf{x}, t)}{\partial t}=-\operatorname{div}\left(\pi_{\mathrm{E}} \cdot \mathbf{J}_{Z}^{0}\right)+a_{\mathrm{K}, \mathrm{E}} \cdot \underset{s}{R} .
$$

Der Korrektor $\pi_{\mathrm{E}}$ wird über das Zell-Problem der Mikrostruktur berechnet und $a_{\mathrm{K}, \mathrm{E}}$ ist die Fläche pro Volumen des porösen Mediums. Diese Gleichungen müssen nicht mehr in der komplexen Geometrie des porösen Mediums gelöst werden, sondern in einem einfachen Würfel (oder auf einem Intervall in $1 \mathrm{D}$ ), was die numerische Rechenzeit deutlich reduziert.

Aufgrund der spezifischen Kopplung der partiellen Differentialgleichungen ist, selbst im $1 \mathrm{D}$ Fall, eine analytische Lösung des Systems nicht zu finden. Es werden daher numerische Verfahren benutzt, beispielsweise Finite-VolumenMethoden, um die Gleichungen zu lösen und a posteriori die Zellspannung als Funktion des Stromes $I$ zu berechnen. Nach der Validierung an experimentellen Daten der $(I-U)$ Kennlinie können mittels des Modells (computergestützte) Vorhersagen bezüglich veränderter Prozess-Parameter (z. B. Temperatur, Elektrolyt-Konzentration und Zusammensetzung, Stromstärke), neuer Zellaufbauten (z. B. Geometrie

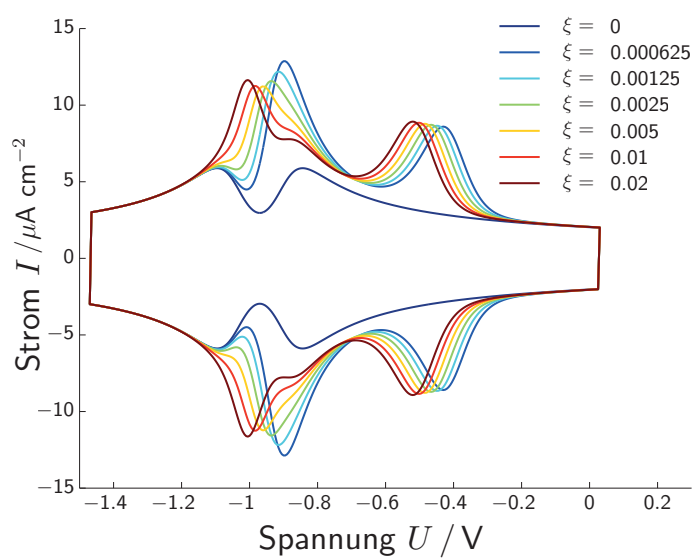

(a) Simulierte Strom-Spannungskurve einer Silber Elektrode in Kontakt mit einer wässrigen Lösung aus $(\xi \mathrm{M} \mathrm{KCl}+$ $\left.(0.2-\xi) \mathrm{MKPF}_{6}\right)$ und Variation der Zusammensetzung $\xi$

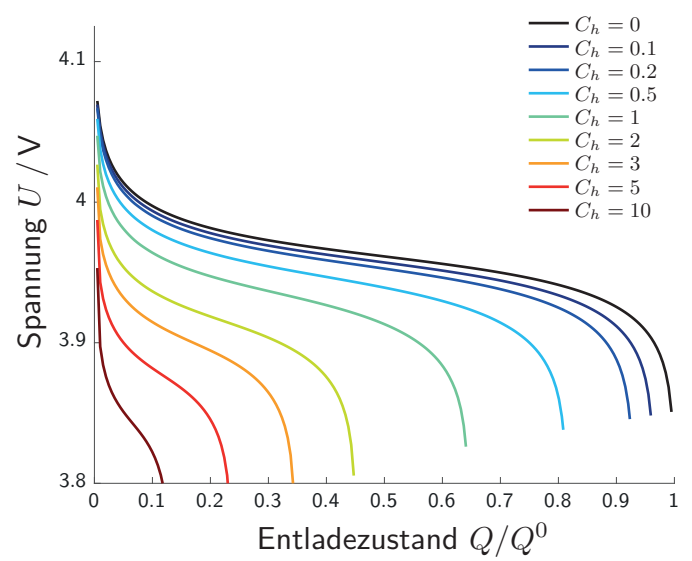

(b) Simulierte Spannung einer Li-Batterie als Funktion des Entladungszustands $Q / Q^{0}\left(Q^{0}\right.$ Kapazität der voll geladenen Batterie) für verschiedene Entladeströme $I=C_{h} \frac{Q^{0}}{60 \mathrm{~min}}$

Abbildung 2. Numerische Berechnung der Strom-Spannungskurven für verschiedene elektrokatalytische Systeme 
der Elektroden, Porosität) oder verschiedener Materialien gemacht werden (siehe Abbildung 2). Die mathematische Modellierung und Simulation ermöglicht dadurch qualitative und quantitative Vorhersagen zum Verhalten von elektrokatalytischen Zellen und trägt damit wesentlich zum Verständnis und zur systematischen Weiterentwicklung bei.
Literatur

[1] J. Newman and K. Thomas. Electrochemical Systems. John Wiley \& Sons, 2014

[2] M. Landstorfer, J. Electrochem. Soc., 2017, 164, 3671-3685

[3] S. de Groot and P. Mazur. Non-Equilibrium Thermodynamics. Dover Publications, 1984

[4] U. Hornung. Homogenization and Porous Media. Springer Science \& Business Media, 2012 der $\mathrm{MATH}^{+}$Application Area 2 - Materials, Light, Devices.

\title{
Mathematik in der Flugplanung
}

\author{
Kamal Al-Bawani, Marco Blanco Sandoval, Ralf Borndörfer, Anton Kaier, \\ Pedro Maristany, Adam Schienle und Swen Schlobach
}

\begin{abstract}
Der Verkehr ist ein Gebiet, in dem die Rolle der Mathematik auf vielfältige Weise sichtbar wird. Autos sind heute mit Routenplanungssystemen ausgestattet, demnächst werden sie autonom fahren. Kein Bahnkunde schaut heute noch im Kursbuch nach einer Verbindung. Internethändler betreiben vollautomatische Hochregallager und schaffen es, Bestellungen am gleichen Tag auszuliefern. In all diesen Systemen wird Mathematik immer mehr nicht nur zur Optimierung genutzt, sie wird selbst zum wesentlichen Bestandteil des Systems, ohne den nichts mehr geht. Das gilt auch für die Planung von Flugrouten.
\end{abstract}

Flugrouten richten sich stark nach dem Wetter: Die Maschinen starten oder landen nicht nur in die Richtung, aus der der Wind weht, auch die restliche Flugroute wird angepasst, um im Jetstream mitzugleiten, um Turbulenzen auszuweichen oder der Aschewolke des Eyjafjallajökull. Auf diese Weise werden Sicherheit und Komfort erhöht, hauptsächlich aber Treibstoff gespart, pro Route bis zu 2,5 Prozent, das ist bei den niedrigen Margen im Luftverkehr sehr viel.

Daher ist die Berechnung des Treibstoffverbrauchs die Basis jeder Routenoptimierung. Prinzipiell verbraucht ein Flugzeug weniger Kerosin, je höher es fliegt, je mehr Rückenwind es hat und je leichter es ist. Insbesondere verringert sich der Verbrauch beim Durchfliegen eines Streckenabschnittes oder "Segmentes“ kontinuierlich. Zur Berechnung geben die Flugzeughersteller für jeden Typ mit einer „specific range" genannten Funktion $f: \mathbb{R}_{+} \rightarrow \mathbb{R}+$ die marginale Reichweite in Abhängigkeit vom aktuellen Gesamtgewicht an (die Höhe und die Temperatur spielen ebenfalls eine Rolle), vgl. Abbildung 1. Deren Stammfunktion

$$
F\left(w_{\text {curr }}\right)=\int_{w_{\text {empty }}}^{w_{\text {curr }}} f(v) \mathrm{dv}
$$

gibt dann die Reichweite an, die das Flugzeug bei einem momentanen Gewicht $w_{\text {curr }}$ hat, bis der Tank beim Gewicht $w_{\text {empty }}$ leer ist. Das Endgewicht $w_{\text {end }}$ eines Flugzeugs beim Durchfliegen eines Segmentes der Länge $d$, und damit der Treibstoffverbrauch, ergibt sich aus der Gleichung

$$
\begin{aligned}
d & =\int_{w_{\text {end }}}^{w_{\text {curr }}} f(v) \mathrm{dv} \\
& =F\left(w_{\text {curr }}\right)-F\left(w_{\text {end }}\right) \Leftrightarrow w_{\text {end }} \\
& =F^{-1}\left(F\left(w_{\text {curr }}\right)-d\right),
\end{aligned}
$$

die man performant und mit kontrolliertem Fehler über eine stückweise Linearisierung der Funktion $F$ löst.

Das Wetter geht in diese Rechnung durch eine Verlängerung oder Verkürzung der Segmentlänge $d$ ein, die das Flugzeug im umgebenden Luftraum über Grund zurücklegt. Da man nicht weiß, zu welchem Zeitpunkt man in ein Segment einfliegt und wie dann das Wetter ist, muss diese Korrektur während der Berechnung „on the fly“ gemacht werden. Dies lässt sich gut mit der zeitlichen Interpolation der Wetterprognose verbinden, die in Abständen von drei Stunden vorliegt. 